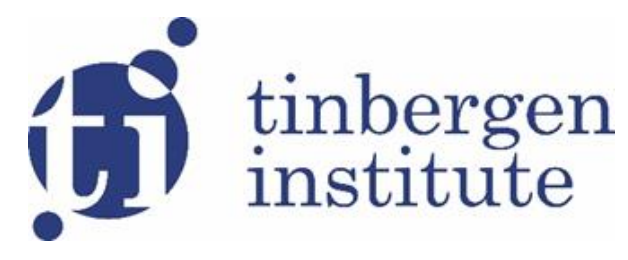

TI 2019-017/VIII

Tinbergen Institute Discussion Paper

\title{
Regulating dynamic congestion externalities with tradable credit schemes: does a unique equilibrium exist?
}

Yue $B a o^{1}$

Erik (E.T.) Verhoef ${ }^{1}$

Paul (P.R.) Koster ${ }^{1}$

${ }^{1}$ VU Amsterdam, The Netherlands 
Tinbergen Institute is the graduate school and research institute in economics of Erasmus University Rotterdam, the University of Amsterdam and VU University Amsterdam.

Contact: discussionpapers@tinbergen.nl

More TI discussion papers can be downloaded at https://www.tinbergen.nl

Tinbergen Institute has two locations:

Tinbergen Institute Amsterdam

Gustav Mahlerplein 117

1082 MS Amsterdam

The Netherlands

Tel.: + 31(0)205984580

Tinbergen Institute Rotterdam

Burg. Oudlaan 50

3062 PA Rotterdam

The Netherlands

Tel.: +31(0)10408 8900 


\title{
Regulating dynamic congestion externalities with tradable credit schemes: does a unique equilibrium exist?
}

\author{
Yue Bao, Erik T. Verhoef*, Paul Koster \\ Department of Spatial Economics, VU University Amsterdam, Amsterdam, the Netherlands
}

\begin{abstract}
Tradable credit schemes offer a potentially efficient, revenue-neutral policy alternative to classical dynamic pricing of congestion externalities. We show in this paper that the resulting equilibrium may not be unique for particular models of congestion, including the first-best solution for the conventional Vickrey's bottleneck model. This can have substantial detrimental impacts on social welfare and social acceptance of tradable credit schemes. The reason underlying this result is that the credit supply-demand condition can be satisfied for a continuum of credit prices. This is because any marginal change in the credit price will be matched by a compensating change in queuing times, keeping user price fixed but deviating from the first-best optimum in which no queueing should occur. We find that the problem of non-uniqueness does not occur for the well-known dynamic flow congestion model proposed by Chu. A unique equilibrium can be obtained in the bottleneck model if the buying and selling of credits with a bank is allowed, against a pre-determined price. Credits are then still tradable so that the use can deviate from the initial distribution, but the credit price is determined by the perfectly elastic demand and supply from the bank.
\end{abstract}

\section{Introduction}

Congestion in urban areas remains a substantial problem worldwide. The estimated costs from congestion between Britain, Germany and the United States are in total \$461billion in 2017, or \$975 per person (The Economist, 2018) while the estimated direct congestion cost in Beijing is 4013.31 Yuan (about \$594) per person in 2017 (China Urban Research Report 2017, 2018). Congestion is inherently dynamic and is governed by daily choices of individuals who make a tradeoff between longer travel times and arriving earlier or later at their destination (e.g., Vickrey, 1969). With limited road capacities and concentrated demand in time and space, traffic congestion around the most preferred arrival times can be severe and as a result most of the commuters will arrive earlier or later than their preferred arrival time at equilibrium. As road users increase delays for others, negative congestion externalities arise and the resulting equilibrium is not socially optimal. Because departure time choices are an important margin of travelers' behavioral adjustment, it is essential to investigate commuters' departure time responses to traffic policies aimed at alleviating rush hour congestion.

A large amount of literature investigated the morning commute congestion problem using dynamic equilibrium models with endogenous departure times (e.g., Hendrickson and Kocur, 1981; Arnott et al., 1990; Laih, 1994; Chu, 1995; Liu and Nie, 2011). Different types of congestion technologies to model the impact of commuters' departure time choices on equilibrium travel time patterns have been developed,

\footnotetext{
*Corresponding author. Email: e.t.verhoef@vu.nl (E.T., Verhoef).
} 
including the well-known bottleneck model (e.g., Vickrey, 1969), and various flow congestion models (e.g., Henderson, 1974, 1977; Chu, 1995; Verhoef, 2017). The pure bottleneck model treats congestion as a spaceless queue behind a single bottleneck, while the flow congestion model applies a speed-flow relation to the entire link, accounting for the length of the road along which congestion is encountered. The bottleneck model of Vickrey (1969) assumes equal capacity over the rush hour, and on the demand side assumes a piece-wise linear marginal utility function for schedule-delay costs (Small and Verhoef, 2007). There are many extensions to Vickrey's model, including models that allow for heterogeneity of preferences, and different types of scheduling preferences (Fosgerau and Small, 2013; van den Berg and Verhoef, 2011). For flow congestion models, differences in model approaches arise from the specification of the dynamic travel time function which provides a structural relationship between capacity, the number of travelers at a certain time instant, and the speed or travel time. Henderson (1974), for instance, applies a formulation where the travel time is determined by the flow at the road's entrance when departing from home, while Chu (1995) uses a comparable specification with the differences that including, travel time is determined by the flow at the road's exit when arriving at work.

Various possible traffic policies to address congestion have been investigated besides road pricing, rewarding (e.g., Rouwendal et al., 2012) and tradable credits (e.g., Yang and Wang, 2011). Practical implementations of road pricing has been discussed for several decades now, and have been implemented in a few cities including London (Transport for London, 2018) and Stockholm (Maria et al., 2012). In theory, it can achieve the system optimum (SO) by internalizing the externalities that drivers impose upon one another. However, public acceptance is low (Verhoef et al., 1997b; Rouwendal et al., 2012) mainly due to the large financial transfers from the public to the government. Rewarding, on the other hand, seems much more acceptable to the public (at least for the road users themselves); however, it poses great budgetary pressure on the government, and is therefore usually financially unsustainable in the long run. As a downside, it is viewed as indefensible as people choosing other modes are not rewarded and in the long run it has the possibility to induce latent travel demand impacts as the price for a trip is decreased (Rouwendal et al., 2012).

In contrast, tradable credits have the advantage of being revenue-neutral in aggregate terms as there is no money flow between road users and the government (Verhoef et al., 1997a): drivers sell and buy credits in the market implying that there is no financial transfer in aggregate terms between drivers and the regulators. Furthermore, theoretically speaking, tradable credit schemes have been proven to generate a high efficiency (e.g., Yang and Wang, 2011 and Wang et al., 2012 for general transportation network; Wu et al., 2012 for multimodal transportation networks; Nie and Yin, 2013 and Tian et al., 2013 for bottleneck models). Due to these potential advantages, there is a growing body of research on tradable credits in recent years (e.g., Verhoef et al., 1997; Raux, 2004; Nie, 2012; Miralinaghi and Peeta, 2016; Bao et al., 2017). More detailed recent reviews can be found in Grant-Muller and Xu (2014), and Dogterom et al., (2016).

Using stylized models, it has been proven that both first-best road pricing and optimal tradable credit schemes result in the system optimum (Xiao et al., 2013). For tradable credit schemes, a time-varying credit 
charge function $(\kappa(t)$ ) needs to be implemented that determines the number of credits needed for an arrival at any given moment. However, we will show that, in contrast to the case of first-best road pricing, the equilibrium outcome when a tradable credit scheme is implemented and the classical bottleneck model is used, is in fact uncertain. Under first-best dynamic pricing in the classic bottleneck model, at any moment a toll applies that is proportional to the length of the queue that would have existed without pricing, and thus travel time delays will be totally eliminated (Arnott et al., 1990). The departure rate during the whole peak period will then be equal to the capacity of the bottleneck, and the system optimum without queuing is the unique equilibrium. We will demonstrate that the resulting first-best equilibrium of the bottleneck model with a tradable credit scheme is not unique, which means that although the first-best system optimum may still be the outcome, so may be a continuum of other, non-optimal equilibria. The implication is that the welfare gains of a tradable credit scheme are uncertain, and are, in expected terms, somewhere in between the unregulated and the first-best outcome.

In particular, for the socially optimal tradable credit scheme, there exists an optimal credit price such that the credit payment is at any moment equal to the difference between the equilibrium generalized travel cost and the schedule-delay cost. However, this credit price is not unique. In fact, for any positive credit price below the optimal price, a queue develops at equilibrium, such that the sum of the queuing delay cost and the credit payment at any time instant is equal to what the credit payment would have been in the optimal case. In this way, the generalized price of travel at each time instant is always equal to the optimal equilibrium generalized price, which means the corresponding departure profile is an equilibrium as well. In other words, every feasible credit price can lead to an equilibrium. Therefore, there is a continuum of equilibria, bounded by the classic no-toll equilibrium and the classic first-best optimum. Uncertain outcomes of the tradable credit schemes, varying between the no-toll equilibrium and the first-best optimum, are likely to be unacceptable for regulators, not in the least place because the welfare gains from the policy will be highly uncertain. Besides this, it is uncertain how traffic patterns are affected. These two factors can raise public questioning of government's abilities to control traffic, reducing the acceptance of the policy.

This paper investigates the non-unique equilibrium of the bottleneck model with optimal tradable credit schemes and will also try to get new insights into how to secure a model with a unique equilibrium outcome. First, the non-uniqueness of equilibrium in the Vickrey's bottleneck model with optimal fine and coarse tradable credit charge schemes is formally demonstrated and discussed. By analyzing non-uniqueness in Vickrey's model in a formal way, we gain insights into the properties of the bottleneck model with optimal tradable credit schemes, as well as the underlying causes of having multiple equilibria. This helps us to find measures to avoid uncertain outcomes, and to provide novel qualitative policy recommendations to regulators. Second, we investigate whether the same problem prevails for a different kind of congestion technology, namely the flow congestion model as proposed by Chu (1995). We show that when Chu's model is used for the analysis of tradable credit schemes, a unique solution is obtained. The empirical implication is that for real traffic systems resembling Chu's model the problem of uniqueness does likely not occur. 
The paper is organized as below. Section 2 analyzes the bottleneck model with a regulator applying a tradable credit schemes. Section 3 analyzes Chu's model with an optimal tradable credit scheme. Section 4 compares the two models and provides conclusions and policy implications.

\section{Optimal tradable credit scheme and the bottleneck model}

Let us consider the basic bottleneck model with a total number of $N$ homogeneous cars passing a bottleneck with given capacity $s$. For convenience and without loss of generality, we set the free-flow travel time to zero. Therefore, in the absence of queuing, cars depart from home, pass the bottleneck and arrive at work at the same moment. When the departure rate $r_{D}$ exceeds the capacity of the bottleneck $s$, a queue develops. The travel time, $T_{q}(t)$, of a driver departing at time $t$, depends on the length of the queue at that moment, and is given by:

$$
T_{q}(t)=\frac{\int_{t_{s}}^{t} r_{D}(\omega) d \omega-s\left(t-t_{s}\right)}{s}
$$

where $t_{s}$ denotes the starting time of the peak period, and we have assumed (as is true at equilibrium) that the outflow of the bottleneck does not fall below $s$ between $t_{s}$ and any relevant moment $t$ at which arrivals occur. Following the standard bottleneck model (e.g., Vickrey, 1969; Arnott et al.,1990), we assume that drivers' trip cost consists of three parts: (1) the travel time cost; (2) the schedule-delay cost; and (3) the road toll or credit payment, when applicable. Denote $t_{e}$ as the end time of the peak period, satisfying $t_{e}=t_{s}+N / s$, the generalized trip cost of a driver departing at time $t \in\left[t_{s}, t_{e}\right]$ is given by:

$$
p(t)=\alpha T_{q}(t)+\beta \cdot \max \left\{0, t^{*}-t-T_{q}(t)\right\}+\gamma \cdot \max \left\{0, t+T_{q}(t)-t^{*}\right\}+\tau\left(t+T_{q}(t)\right)
$$

where $\alpha$ is the value of time, $\beta$ is the unit schedule early cost, and $\gamma$ is the unit schedule late cost where it is normally assumed that $\beta<\alpha<\gamma^{1}$. $\tau(\cdot)$ denotes the road toll/credit payment defined as a function of arrival time. Furthermore, we define $\delta \equiv \beta \cdot \gamma /(\beta+\gamma)$ as a schedule-delay cost parameter.

According to Arnott et al. (1990), the social optimum can be decentralized by the following time-dependent road toll (the optimal fine toll):

$$
\tau\left(t^{\prime}\right)= \begin{cases}a-\beta\left(t^{*}-t^{\prime}\right), & t^{\prime} \in\left[t_{s}^{\mathrm{so}}, t^{*}\right) \\ a-\gamma\left(t^{\prime}-t^{*}\right), & t^{\prime} \in\left[t^{*}, t_{e}^{\mathrm{so}}\right]\end{cases}
$$

where $a=\delta N / s$ if the toll starts and ends at a zero level and $t^{\prime}=t+T_{q}(t)$ denotes the arrival time at the bottleneck exit (or at the destination). Given the above optimal fine toll, the equilibrium departure rate is equal to the bottleneck capacity $s$, and no queuing delay exists. The total schedule-delay is the same as in the no-toll equilibrium, with $t_{s}^{\text {so }}=t^{*}-\gamma /(\beta+\gamma) \cdot N / s$ and $t_{e}^{\text {so }}=t^{*}+\beta /(\beta+\gamma) \cdot N / s$.

\footnotetext{
${ }^{1} \beta<\alpha$ is adopted to secure an equilibrium without mass departures, while $\alpha<\gamma$ is often found empirically but is not required to secure equilibrium.
} 
In a similar vein, the optimal fine time-dependent credit charge rate function can reach the SO equilibrium (see Xiao et al., 2013). For convenience, we assume the credit charge at $t_{s}$ is zero (which is a harmless assumption with perfectly inelastic demand), and consider the following credit charge rate:

$$
\kappa\left(t^{\prime}\right)= \begin{cases}\frac{\bar{a}}{\eta}-\frac{\beta}{\eta}\left(t^{*}-t^{\prime}\right), & t^{\prime} \in\left[t_{s}^{\mathrm{so}}, t^{*}\right) \\ \frac{\bar{a}}{\eta}-\frac{\gamma}{\eta}\left(t^{\prime}-t^{*}\right), & t^{\prime} \in\left[t^{*}, t_{e}^{\mathrm{so}}\right]\end{cases}
$$

where $\bar{a}=\delta N / s$, and $\eta>0$ is a constant to be chosen. The generalized price of a driver departing at time $t \in\left[t_{s}^{\mathrm{so}}, t_{e}^{\mathrm{so}}\right]$ under the above credit charge rate Eq. (4) is then given by:

$$
p(t)=\alpha T_{q}(t)+\rho \frac{\bar{a}}{\eta}-\max \left\{\rho \frac{\beta}{\eta}\left(t^{*}-t^{\prime}\right), \rho \frac{\gamma}{\eta}\left(t^{\prime}-t^{*}\right)\right\}+\max \left\{\beta\left(t^{*}-t^{\prime}\right), \gamma\left(t^{\prime}-t^{*}\right)\right\}
$$

where $\rho \geq 0$ is the endogenous credit price. When the credit price is positive, a credit market clearing condition must hold at equilibrium: the number of credits paid should be equal to the number of credits issued, K. With Eq. (4), we have:

$$
\mathrm{K}=\int_{t_{s}^{50}}^{t_{e}^{50}} S \kappa\left(t^{\prime}\right) d t^{\prime}=\frac{\bar{a} N}{2 \eta}
$$

If the endogenous credit price, $\rho$, is equal to $\eta$, the credit payment is given by:

$$
\tau\left(t^{\prime}\right)= \begin{cases}a-\beta\left(t^{*}-t^{\prime}\right), & t^{\prime} \in\left[t_{s}^{\text {so }}, t^{*}\right) \\ a-\gamma\left(t^{\prime}-t^{*}\right), & t^{\prime} \in\left[t^{*}, t_{e}^{\text {so }}\right]\end{cases}
$$

In this case, the SO equilibrium will be decentralized by the above credit charge $\kappa\left(t^{\prime}\right)$ and the equilibrium generalized price is given by:

$$
p^{\mathrm{so}}=\beta\left(t^{*}-t_{s}^{\mathrm{so}}\right)=\gamma\left(t_{e}^{\mathrm{so}}-t^{*}\right)
$$

which is indeed the same with the socially optimal price. However, different from the optimal fine toll, the credit payment also depends on the endogenous equilibrium credit price. Condition (6) shows that the credit demand is independent of the credit price. Therefore, for any departure time $t \in\left[t_{s}^{\mathrm{so}}, t_{e}^{\mathrm{so}}\right]$, as long as the sum of queuing delay cost $\alpha T_{q}(t)$ and credit payment $\rho \kappa\left(t^{\prime}\right)$ equals the difference of the generalized trip cost and the schedule-delay cost, the resulting departure profile is an equilibrium departure profile. More specifically, given a credit price $0 \leq \rho<\eta$, any departure rate $r_{D}(t)$ satisfying the following condition is an equilibrium departure rate:

$$
\alpha T_{q}(t)+\rho \frac{\bar{a}}{\eta}-\max \left\{\rho \frac{\beta}{\eta}\left(t^{*}-t^{\prime}\right), \rho \frac{\gamma}{\eta}\left(t^{\prime}-t^{*}\right)\right\}=\beta\left(t^{*}-t_{s}^{\mathrm{so}}\right)-\max \left\{\beta\left(t^{*}-t^{\prime}\right), \gamma\left(t^{\prime}-t^{*}\right)\right\}
$$

Because $T_{q}(t)$ and $\kappa\left(t^{\prime}\right)$ are, at equilibrium, both zero at $t_{s}^{\text {so }}$ and $t_{e}^{\text {so }}$, their sum moves piecewise-linearly with the interval $t \in\left[t_{s}^{\text {so }}, t_{e}^{\text {so }}\right]$. Because $\kappa\left(t^{\prime}\right)$ is piecewise-linear in that same interval, a continuum of 
equilibria exist for the given credit charge rate (4). Any credit price $\rho$ between 0 and $\eta$ can be matched with a triangular travel delay cost pattern $T_{q}(t)$ that has its top at $t^{*}$, at any level between 0 and $a$.

Figure 1 shows two equilibria of the bottleneck model under the optimal fine tradable credit scheme: the SO equilibrium, and an (arbitrarily) chosen non-optimal equilibrium. Figure 1(a) gives the cumulative departures of these two equilibria. The blue line shows the SO case with $\rho=\rho^{\text {so }}=\eta$ for which the departure rate equals the bottleneck capacity. As a result, no queue exists and the queuing delay is zero. The red line shows the cumulative departures in the non-optimal equilibrium case when the credit price $0<\rho^{\prime}<\eta$. The departure rate for early departures is larger than the bottleneck capacity and a queue of time-varying length exists during the whole peak period (except the endpoints). The queuing delay follows the familiar triangular pattern that is also observed in the standard no-toll equilibrium, but with a lower top for positive credit prices. Figure 1(b) presents the corresponding credit payments for the SO case (blue line) and the non-optimal case (red line). For the SO case, the queuing time is zero, as the departure rate is equal to the bottleneck capacity throughout the peak. In this case, the generalized price consists of two parts: the schedule-delay costs and the credit payment with the credit price $\rho^{\text {so }}$. On the other hand, in the non-optimal case (with the credit price $\rho^{\prime}<\rho^{\text {so }}$ ), the credit payment is less than that in the SO case. As a result, a queue develops and the queuing delay cost is equal to the difference in credit payments between the SO case and the non-optimal case. The generalized price will then be constant over arrival times between $t_{s}^{\mathrm{so}}$ and $t_{e}^{\mathrm{so}}$, confirming it is also an equilibrium.
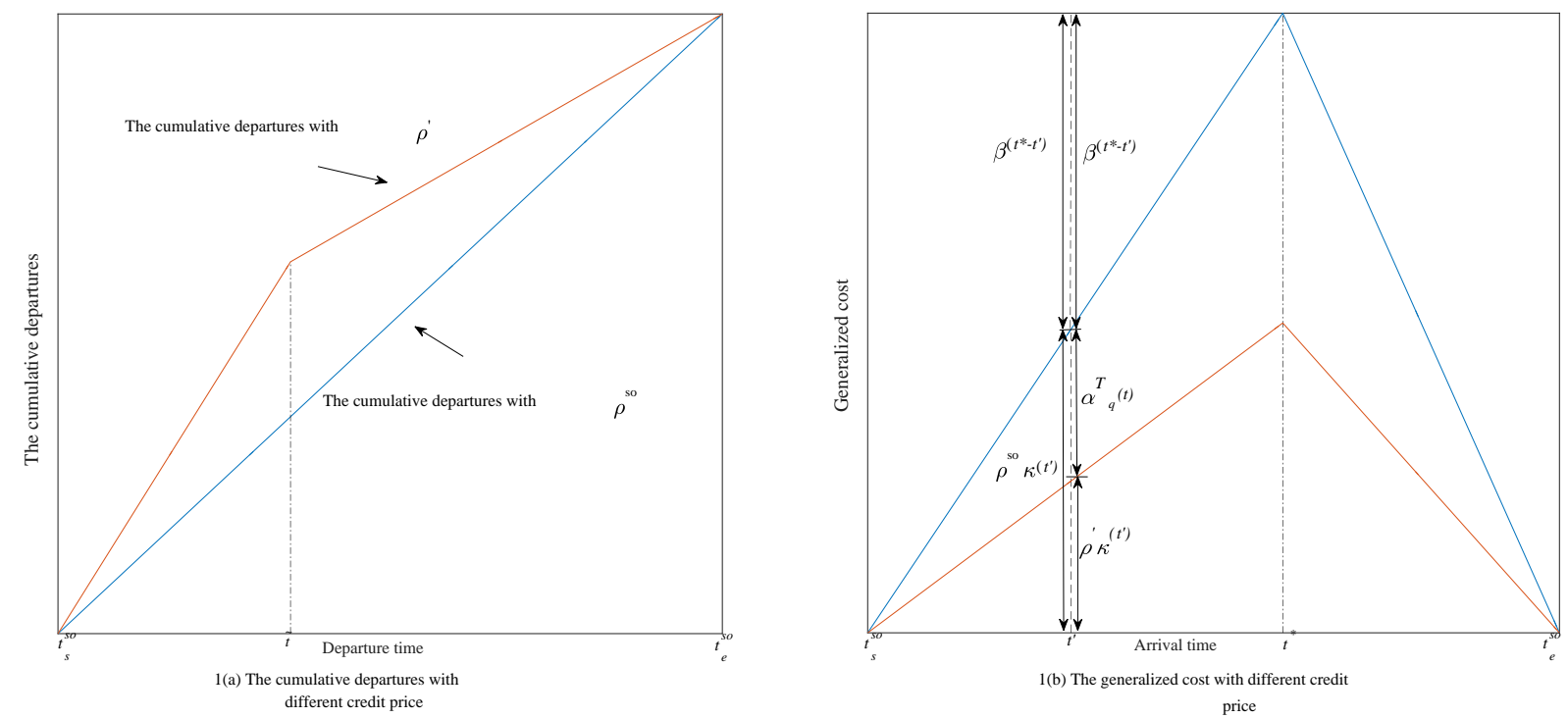

Figure 1. The trip costs and the cumulative departures with fine credit charge

The non-uniqueness of equilibrium is a sharp contrast with the uniqueness that applies for classic road pricing, and is caused by the fact that the credit market clearing condition (Eq. 6) is satisfied without a unique constraint on the credit price. Different feasible credit prices subsequently result in multiple equilibria. It is important to note that this type of non-uniqueness also applies for the optimal step credit 
charge scheme (see Appendix 1). Therefore, first-best and second-best optimal credit charge schemes cannot ensure the achievement of the equilibria obtained with the corresponding pricing variants of the same policies. There is a relatively easy solution to this problem of non-uniqueness, that maintains the credit-trading policy's attractive properties of being budget neutral and securing an efficient allocation. That is to fix the credit price $\rho$, to a value of $\rho^{\text {so }}$ (1, in our previous example). If the optimal number of credits is distributed for free ("grandfathered"), there will be no buying from or selling to the bank that executes the perfectly elastic supply or demand for credits, but the mere possibility to do so secures that the market price will be $\rho^{\text {so }}$, and the intended optimum will indeed be attained, while it may well involve trade between travelers before equilibrium is established ${ }^{2}$.

\section{Flow congestion and tradable credit schemes}

Given that the optimal tradable credit scheme cannot ensure a unique equilibrium departure profile in the bottleneck model, a next question is whether with a different congestion technology, a unique social optimal equilibrium with tradable credits is found. This section shows that this may indeed be the case, using the flow congestion technology proposed by Chu (1995). Even though it is a rather stylized model, it provides an important extension to the bottleneck model, as the duration of the peak and the generalized price differs between the no-toll equilibrium and the first-best optimum. This is exactly the generalization we seek to consider: the Chu model provides the analytical simplest way of doing that. It assumes that the travel time only depends on the arrival rate (at the destination) at the moment the trip is completed.

Given an arrival time $t^{\prime}$ and the arrival rate $r_{A}\left(t^{\prime}\right)$, and with $v\left(r_{A}\left(t^{\prime}\right)\right)$ denoting the travel speed function of the entire trip, for a trip length $m$ the travel time is $m / v\left(r_{A}\left(t^{\prime}\right)\right)$. The travel time for a trip completed at $t^{\prime}$ thus depends on the free-flow speed, $V_{\max }$. Following Chu (1995), we define a BPR-type power function:

$$
\frac{m}{v\left(t^{\prime}\right)}=\frac{m}{V_{\max }}+m\left[\frac{r_{A}\left(t^{\prime}\right)}{R}\right]^{v}=T_{f}+m\left[\frac{r_{A}\left(t^{\prime}\right)}{R}\right]^{v}
$$

where $R$ is the capacity, $v$ a parameter representing the curvature of the congestion function and the free flow travel time as $T_{f}=m / V_{\max }$. The generalized price again includes three parts: the travel time cost, the schedule-delay cost, and the credit payment:

$$
p\left(t^{\prime}\right)=\left\{\begin{array}{l}
\alpha \frac{m}{v\left(t^{\prime}\right)}+\beta\left(t^{*}-t^{\prime}\right)+\rho \kappa\left(t^{\prime}\right), t_{s} \leq t^{\prime} \leq t^{*} \\
\alpha \frac{m}{v\left(t^{\prime}\right)}+\gamma\left(t^{\prime}-t^{*}\right)+\rho \kappa\left(t^{\prime}\right), t^{*}<t^{\prime} \leq t_{e}
\end{array}\right.
$$

\footnotetext{
${ }^{2}$ Note that the resulting situation would not be equivalent to classic road pricing. That would only be true if credits were sold instead of grandfathered and would not be tradable.
} 
In the equilibrium with optimal pricing, those arriving at $t_{s}$ or $t_{e}$ incur no travel delay cost and no toll (under classic road pricing) or credit payment (with tradable credits). Then, the trip costs at $t_{s}$ and $t_{e}$ are given by:

$$
\begin{aligned}
& p\left(t_{s}\right)=\alpha T_{f}+\beta\left(t^{*}-t_{s}\right) \\
& p\left(t_{e}\right)=\alpha T_{f}+\gamma\left(t_{e}-t^{*}\right)
\end{aligned}
$$

As every driver has the same generalized price, setting the trip cost in Eq. (11) equal to Eq. (12) for $t^{\prime}<t^{*}$ and equal to Eq. (13) for $t^{\prime}>t^{*}$ yields the equilibrium travel time function:

$$
\frac{m}{v\left(t^{\prime}\right)}=\left\{\begin{array}{l}
T_{f}+\frac{\beta}{\alpha}\left(t^{\prime}-t_{s}\right)-\frac{\rho}{\alpha} \kappa\left(t^{\prime}\right), t_{s} \leq t^{\prime} \leq t^{*} \\
T_{f}+\frac{\gamma}{\alpha}\left(t_{e}-t^{\prime}\right)-\frac{\rho}{\alpha} \kappa\left(t^{\prime}\right), t^{*} \leq t^{\prime} \leq t_{e}
\end{array}\right.
$$

The other conditions that must be satisfied include that the price at $t_{s}$ and $t_{e}$ must be equal, and that the cumulative arrivals between $t_{s}$ and $t_{e}$ equal the number of drivers, i.e.:

$$
\begin{gathered}
p\left(t_{s}\right)=p\left(t_{e}\right) \\
\int_{t_{s}}^{t_{e}} r_{A}\left(t^{\prime}\right) d t^{\prime}=N
\end{gathered}
$$

The credit market clearing condition can now be written as a complementary slackness condition:

$$
\rho\left(\mathrm{K}-\int_{t_{s}}^{t_{e}} r_{A}\left(t^{\prime}\right) \kappa\left(t^{\prime}\right) d t^{\prime}\right)=0 ; \rho \geq 0 ; \mathrm{K}-\int_{t_{s}}^{t_{e}} r_{A}\left(t^{\prime}\right) \kappa\left(t^{\prime}\right) d t^{\prime} \geq 0
$$

This shows that either the number of credits consumed equals the number of credits issued, or the credit price will be zero.

Following the optimal toll scheme in Chu (1995), the optimal credit charge rate is given by (18), with the optimal credit price $\rho^{0}=1$ reproducing the first-best time-varying toll

and the amount of credit issued should be equal to:

$$
\kappa^{\mathrm{o}}\left(t^{\prime}\right)=\left\{\begin{array}{l}
\frac{v \beta}{1+v}\left(t^{\prime}-t_{s}^{\mathrm{o}}\right), t_{s}^{\mathrm{o}} \leq t^{\prime} \leq t^{*}, \\
\frac{v \gamma}{1+v}\left(t_{e}^{\mathrm{o}}-t^{\prime}\right), t^{*}<t \leq t_{e}^{\mathrm{o}}
\end{array}\right.
$$

$$
\mathrm{K}^{\mathrm{o}}=\alpha \frac{\mathrm{v}(1+\mathrm{v})^{\frac{1}{1+\mathrm{v}}}}{(2 \mathrm{v}+1)} \Psi N
$$

which equals the toll revenues given in Chu (1995)'s original paper. The optimal travel time function (as a function of clock time) is also given by Chu (1995) and reads

$$
\frac{m}{v^{0}\left(t^{\prime}\right)}=\left\{\begin{array}{l}
T_{f}+\frac{1}{1+v} \frac{\beta}{\alpha}\left(t^{\prime}-t_{s}^{0}\right), t_{s}^{0} \leq t^{\prime} \leq t^{*} \\
T_{f}+\frac{1}{1+v} \frac{\gamma}{\alpha}\left(t_{e}^{0}-t^{\prime}\right), t^{*} \leq t^{\prime} \leq t_{e}^{o}
\end{array}\right.
$$


where $t_{s}^{0}=t^{*}-\alpha / \beta \Psi(1+v)^{1 /(1+v)}, t_{e}^{0}=t^{*}+\alpha / \gamma \Psi(1+v)^{1 /(1+v)}$ and $\Psi=\left(\frac{N}{R} \frac{1+v}{v} \frac{\beta \gamma}{\alpha(\beta+\gamma)} m^{1 / v}\right)^{v /(1+v)}$. The resulting numbers of early departures and late departures are $\gamma /(\gamma+\beta) N$ and $\beta /(\gamma+\beta) N$, respectively; the same ratio as in Vickrey's bottleneck model.

We now turn to the key question: are there also multiple equilibria for this congestion technology when the optimal tradable credit scheme is implemented? To answer this question, let us assume that there exists another pair of $\hat{\rho}, \hat{t}_{s}, \hat{t}_{e}$ and $m / \hat{v}\left(t^{\prime}\right)$ such that the above conditions (15)-(17) hold under the tradable credit scheme in Eqs. (18)-(19), where at least one of $\hat{t}_{s}, \hat{t}_{e}$ and $m / \hat{v}\left(t^{\prime}\right)$ has a different value from that in the optimal case. Based on the relationships between $\hat{t}_{s}$ and $t_{s}^{\mathrm{o}}, \hat{t}_{e}$ and $t_{e}^{\mathrm{o}}$, there are four kinds of peak periods different from the optimal peak period $\left[t_{s}^{0}, t_{e}^{0}\right]$, i.e., $\hat{t}_{s}<t_{s}^{\mathrm{o}}$ and $\hat{t}_{e}>t_{e}^{\mathrm{o}}, \hat{t}_{s}>t_{s}^{\mathrm{o}}$ and $\hat{t}_{e}<t_{e}^{\mathrm{o}}, \hat{t}_{s}<t_{s}^{\mathrm{o}}$ and $\hat{t}_{e}<t_{e}^{\circ}, \hat{t}_{s}>t_{s}^{\circ}$ and $\hat{t}_{e}>t_{e}^{0}$. Before analyzing the uniqueness of the equilibrium, we first give the following lemma.

Lemma 1. For any possible equilibrium credit price $\rho$ with $\rho \geq 0$ under the tradable credit scheme given by Eqs. (18)-(19), 1). the numbers of early and late arrivals are fixed, and equal those in the optimal case, 2) the peak periods $\left[\hat{t}_{s}, \hat{t}_{e}\right]$ that have $\left\{\hat{t}_{s}<t_{s}^{\mathrm{o}}, \hat{t}_{e}<t_{e}^{\mathrm{o}}\right\}$, or $\left\{\hat{t}_{s}>t_{s}^{\mathrm{o}}, \hat{t}_{e}>t_{e}^{\mathrm{o}}\right\}$ cannot describe an equilibrium.

\section{Proof. See Appendix 2.}

Based on Lemma 1, there are two kinds of possible deviations from SO if $\rho$ would not become optimal, and the numbers of early and late arrivals are always fixed for any possible deviation (Figure 2). If the credit price is higher than that in the optimal case, some drivers will be pushed out of the optimal peak period $\left[t_{s}^{0}, t_{e}^{0}\right]$, and $t_{s}<t_{s}^{o}$ and $t_{e}>t_{e}^{o}$ hold for the resulting equilibrium. Reversely, if the credit price is lower than that in the optimal case, drivers will be attracted to the middle of the optimal peak period closer to the arrival pattern in the no-toll equilibrium, and $t_{s}>t_{s}^{0}$ and $t_{e}<t_{e}^{0}$ hold for the resulting equilibrium. We first consider the case $t_{s}<t_{s}^{0}$ and $t_{e}>t_{e}^{0}$, corresponding with a permit price that has become higher than optimal. Recall that the credit charge function $\kappa^{0}\left(t^{\prime}\right)$ in Eq. (18) is defined using the optimal $t_{s}^{0}$ and $t_{e}^{0}$. Because, as illustrated in Figure 2, a certain share of drivers arrives outside the interval where credits are needed $\left(\left[t_{s}^{0}, t_{e}^{0}\right]\right)$, and, moreover, the density of intervals in the interval where many credits are needed declines, it is intuitive that the number of credits used before $t^{*}$ declines compared to the case with an optimal $\rho$. The same occurs, by symmetry, during late arrivals. The intuition can be substantiated by checking the arrival rate. Focusing on the first part of the peak, where $N \cdot \gamma /(\gamma+\beta)$ arrivals occur, the higher charge $\rho \kappa^{0}\left(t^{\prime}\right)$ means that the arrival rate $r_{A}\left(t^{\prime}\right)$ will display a flatter pattern in the interval $\left[t_{s}^{0}, t^{*}\right)$ 
(see Appendix 3). This, in turn, means that if the $N \cdot \gamma /(\gamma+\beta)$ arrivals maintain their order, each of them except for the person arriving at $t^{*}$ will arrive at a moment that requires a lower number of credits, so that the total demand is smaller than what is consistent with the optimum. The same applies for late arrivals. As a consequence, the demand for credit will fall short of the supply, and the price will fall to zero, which is inconsistent with $\rho>\rho^{\circ}=1$. Similarly, when $\rho<\rho^{\circ}=1$, arrivals will occur in a smaller interval than $\left[t_{s}^{0}, t_{e}^{0}\right]$. Now the arrival rate for $\rho$ will always be steeper than that for $\rho^{\circ}=1$, while both obtain the same value $N \cdot \gamma /(\gamma+\beta)$ at $t^{*}$. Every individual except for the person at $t^{*}$ will now arrive at a moment that requires a higher number of credits if everybody maintains order, so credit demand exceeds supply. This means that $\rho$ will rise as long as $\rho<\rho^{\circ}=1$, confirming that only $\rho^{\circ}$ will be an equilibrium price.

Based on the above analyses, we have the following proposition:

Proposition 1. Given the optimal fine tradable credit scheme in (18)-(19), the congestion technology adopted in Chu's model will have a unique equilibrium if the optimal credit charge function is set and the corresponding optimal number of credits is issued.

Proof. See above and Appendix 3.
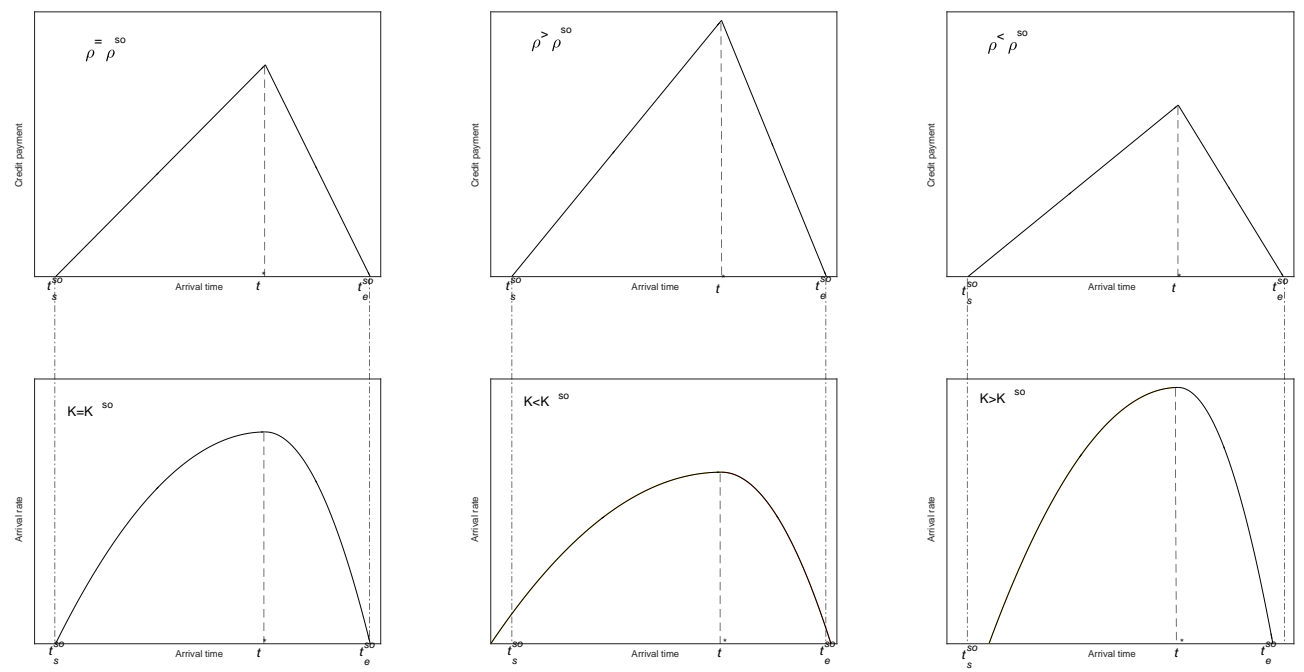

Figure 2. The possible equilibria in Chu's model under optimal tradable credit scheme

From the above analyses, we find that different congestion technologies result in different uniqueness properties of the equilibria. These follow from the relationships between the credit demand, the departure profile and the credit price. In Vickrey's bottleneck model, the arrival rate depends on the capacity of the bottleneck, which is fixed, while the arrival rate in Chu's model is a function of arrival time and the credit price. From the credit market clearing condition (Eq. (17)), a positive credit price requires that the total amount of credits should be equal to the amount of credits issued by the government. For Chu's model, the 
demand for credits is uniquely determined by the arrival rate function and the peak period, both of which are uniquely determined by the credit price. However, the demand for credits in Vickrey's bottleneck model in Section 2 are independent of the credit price. For the fine credit charge scheme case, given the tradable credit scheme, the credit demand only depends on the beginning time of the peak period, which is independent of the credit price. Therefore, the credit market clearing condition is satisfied without any constraints on the credit price, which leads to the existence of multiple equilibria with the same peak period but different departure rate functions. In the coarse credit charge scheme case, given the credit charge scheme, the demand of the credit is fixed and independent of the credit price and peak period. As a result, the credit market clearing condition holds without any constraints on both the credit price and the peak period, and thus, multiple equilibria with different peak periods and departure rate functions exist. Although not considered in this section, it is easily demonstrated that a unique equilibrium exists in Chu's model also under the coarse credit charge scheme, as the credit demand still depends on the arrival rate function, and the arrival rate function is still uniquely determined by the credit price.

\section{Conclusions and policy implications}

This paper investigates the non-uniqueness in the morning commute problem under the optimal tradable credit scheme. We have demonstrated that there are multiple equilibria (in fact, a continuum) in the bottleneck model under the first-best optimal tradable credit scheme. One might object that this problem might disappear when having separate markets for credits for different moments. However, this in first place ignores that time is continuous, implying that for the optimal time-varying policy a continuum of markets would be needed. Second, we have shown in Appendix 2 that also for a coarse credit scheme (representing a market for a time window of discrete measure), non-uniqueness prevails. Different from the case of optimal road pricing, the monetary cost of credit charge depends not only on the charge rate but also on the equilibrium price of the credit. However, the credit market clearing condition can be satisfied for a continuum of credit prices in the bottleneck model, each of which is consistent with a different equilibrium departure profile.

If the resulting equilibrium is unique, the optimal tradable credit scheme can be a first-best efficient policy. But the uncertainty of the outcomes in the bottleneck model makes it much less ideal. Fortunately, nonuniqueness does not occur in all kinds of morning commute problems. For example, for the flow congestion model of Chu, a unique equilibrium result exists. Crucial for this result is that the density of arrivals varies with the price of the credits, which guarantees a unique equilibrium. If the traffic environment in reality is similar to the flow congestion, optimal tradable credit scheme remains an efficient choice to alleviate the congestion during morning rush hours. Moreover, also for pure bottleneck congestion one can relatively easily solve the non-uniqueness problem by fixing the credit price through a perfectly elastic demand/supply of credits by the "bank".

The difference between the bottleneck model and Chu's model is caused by the different relations between credit demand and credit price in the two models. Given the credit charge rate function, credit demand depends on the arrival rate function and the endpoints of the peak period. In Vickrey's model, the arrival

rate function is fixed and equals the capacity of the bottleneck. Thus, credit demand is only determined by 
the duration of the peak period. However, the peak period leading to the optimal credit demand can be obtained for any positive credit price not exceeding the optimal one. Therefore, the credit supply-demand condition can be satisfied by a continuum of credit prices, all of which lead to different equilibrium departure rate functions with the same peak period. On the other hand, in Chu's model, the arrival rates, and the corresponding peak duration vary with the credit price. As a result, the credit demand monotonously decreases with the credit price, which means there exists a unique credit price satisfying the credit supplydemand condition, and a corresponding unique equilibrium departure rate profile exists. Table 1 summarizes our results.

Table 1. Comparisons between Chu's model and Vickrey’s model under tradable credit scheme

\begin{tabular}{|c|c|c|}
\hline & Vickrey’s model & Chu's model \\
\hline Problem & \multicolumn{2}{|c|}{ A fixed number of commuters travel on the same road with the same preferred arrival time at destination } \\
\hline $\begin{array}{l}\text { Congestion } \\
\text { technology }\end{array}$ & Bottleneck approach & Flow congestion approach \\
\hline Assumptions & $\begin{array}{l}\text { Travel time is equal to the free-flow travel time plus the } \\
\text { queuing time at the bottleneck; the arrival rate is equal to } \\
\text { the fixed capacity of the bottleneck; the duration of the } \\
\text { peak period is fixed }\end{array}$ & $\begin{array}{l}\text { Travel time depends on the free-flow } \\
\text { travel time and the flow one arrives; the } \\
\text { arrival rate is a function of time; the } \\
\text { duration of the peak period is uncertain }\end{array}$ \\
\hline Suitable situation & Queuing situations & Flow congestion \\
\hline Measure & Optimal fine tradable credit scheme & Optimal fine tradable credit scheme \\
\hline Outcome & Multiple equilibria & Unique equilibrium \\
\hline Peak period & Fixed length & Variable length \\
\hline $\begin{array}{l}\text { Equilibrium credit } \\
\text { demand }\end{array}$ & Depending only on the duration of peak period & $\begin{array}{l}\text { Uniquely determined by the peak period } \\
\text { and the arrival rate }\end{array}$ \\
\hline Credit price & Any positive value with $0<\rho \leq \rho^{\text {so }}$ & $\begin{array}{l}\text { Uniquely determined by the peak period } \\
\text { and the arrival rate }\end{array}$ \\
\hline $\begin{array}{c}\text { Equilibrium } \\
\text { departure profile }\end{array}$ & Depending on the credit price & Depending on the credit price \\
\hline
\end{tabular}

We reiterate that for the bottleneck model, if the credit price is fixed because of a perfectly elastic demand /supply of credits from a bank a unique SO equilibrium prevails. Furthermore, revenue-neutrality of the scheme can be maintained by a grandfathering of the optimal amount of credits. Finally, it should be noted that inelastic overall travel demand is neither a sufficient nor a necessary reason for the non-uniqueness. It is exactly because, for non-unique equilibria, the generalized price is independent of the credit price, that the analysis remains equally relevant if overall demand becomes price sensitive. Therefore, non-uniqueness still exists (see Appendix 4 for a proof).

\section{References}

Arnott, R., de Palma, A., Lindsey, R., 1990. Economics of a bottleneck. J. Urban Econ. 27, 111-130. doi:10.1016/0094-1190(90)90028-L

Arnott, R., De Palma, A., Lindsey, R., 1988. Schedule Delay and Departure Time Decisions with Heterogeneous Commuters. Transp. Res. Rec. 1197, 56-67.

Bao, Y., Gao, Z., Yang, H., Xu, M., GuangMin, W., 2017. Private financing and mobility management of road network with tradable credits. Transp. Res. Part A Policy Pract. 97, 158-176. doi:10.1016/j.tra.2017.01.013 
Börjesson, M., Eliasson, J., Franklin, J.P., 2012. Valuations of travel time variability in scheduling versus mean-variance models. Transp. Res. Part B Methodol. 46, 855-873. doi:10.1016/j.trb.2012.02.004

China Urban Research Report 2017, Baidu Map, https://huiyan.baidu.com/reports/2017Q4_niandu.html , Jan 23, 2018.

Chu, X., 1995. Endogenous Trip Scheduling: The Henderson Approach Reformulated and Compared with the Vickrey Approach. J. Urban Econ. doi:10.1006/juec.1995.1017

Dogterom, N., Ettema, D., Dijst, M., 2016. Tradable credits for managing car travel: a review of empirical research and relevant behavioural approaches. Transp. Rev. 0, 1-22. doi:10.1080/01441647.2016.1245219

Fosgerau, M., Small, K.A., 2013. Hypercongestion in downtown metropolis. J. Urban Econ. 76, 122-134. doi:10.1016/j.jue.2012.12.004

Grant-Muller, S., Xu, M., 2014. The Role of Tradable Credit Schemes in Road Traffic Congestion Management. Transp. Rev. doi:10.1080/01441647.2014.880754

Henderson, J.V., 1974. Road Congestion A Reconsideration of Pricing Theory. J. Urban Econ. 1, 346365. doi:10.1016/0094-1190(74)90012-6

Hendrickson, C., Kocur, G., 1981. Schedule Delay and Departure Time Decisions in a Deterministic Model. Transp. Sci. 15, 62-77. doi:10.1287/trsc.15.1.62

Laih, C.H., 1994. Queueing at a bottleneck with single- and multi-step tolls. Transp. Res. Part A Policy Pract. 28, 197-208. doi:10.1016/0965-8564(94)90017-5

Maria, B., Eliasson, J., Hugosson, M.B., Brundell-freij, K., 2012. The Stockholm congestion charges - 5 years on . Effects , acceptability and lessons learnt 20, 1-12. doi:10.1016/j.tranpol.2011.11.001

Nie, Y.M., 2012. Transaction costs and tradable mobility credits. Transp. Res. Part B Methodol. 46, 189203. doi:10.1016/j.trb.2011.10.002

Nie, Y.M., Yin, Y., 2013. Managing rush hour travel choices with tradable credit scheme. Transp. Res. Part B Methodol. 50, 1-19. doi:10.1016/j.trb.2013.01.004

Raux, C., 2004. The use of transferable permits in transport policy. Transp. Res. Part D Transp. Environ. 9, 185-197. doi:10.1016/j.trd.2004.01.001

Rouwendal, J., Verhoef, E.T., Knockaert, J., 2012. Give or take? Rewards versus charges for a congested bottleneck. Reg. Sci. Urban Econ. 42, 166-176. doi:10.1016/j.regsciurbeco.2011.08.011

Small, K.A., Verhoef, E.T., 2007. The Economics of Urban Transportation. Routledge, Abingdon, England, Routledge, Abingdon, England.

Tian, L.J., Yang, H., Huang, H.J., 2013. Tradable credit schemes for managing bottleneck congestion and modal split with heterogeneous users. Transp. Res. Part E Logist. Transp. Rev. 54, 1-13. doi:10.1016/j.tre.2013.04.002

The Economist, https://www.economist.com/graphic-detail/2018/02/28/the-hidden-cost-of-congestion, Feb 28, 2018.

Transport for London, https://tfl.gov.uk/corporate/publications-and-reports/congestion-charge, Dec, 2018.

Van den Berg, V., Verhoef, E.T., 2011. Congestion tolling in the bottleneck model with heterogeneous values of time. Transp. Res. Part B Methodol. 45, 60-78. doi:10.1016/j.trb.2010.04.003

Verhoef, E., Nijkamp, P., Rietveld, P., 1997a. Tradeable permits: Their potential in the regulation of road transport externalities. Environ. Plan. B Plan. 24, 527-548. doi:10.1068/b240527

Verhoef, E.T., Nijkamp, P., Rietveld, P., 1997b. The Social Feasibility of Road Pricing : A Case Study for the Randstad Area. J. Transp. Econ. Policy 31, 255-276.

Vickrey, W.S., 1969. Congestion theory and transport investment. Am. Econ. Rev. (Papers Proceedings) 59, 251-260.

Wang, X., Yang, H., Zhu, D., Li, C., 2012. Tradable travel credits for congestion management with heterogeneous users. Transp. Res. Part E Logist. Transp. Rev. 48, 426-437. doi:10.1016/j.tre.2011.10.007

Wu, D., Yin, Y., Lawphongpanich, S., Yang, H., 2012. Design of more equitable congestion pricing and tradable credit schemes for multimodal transportation networks. Transp. Res. Part B Methodol. 46, 1273-1287. doi:10.1016/j.trb.2012.05.004 
Xiao, F., Qian, Z., Zhang, H.M., 2013. Managing bottleneck congestion with tradable credits. Transp.

Res. Part B Methodol. 56, 1-14. doi:10.1016/j.trb.2013.06.016

Yang, H., Wang, X., 2011. Managing network mobility with tradable credits. Transp. Res. Part B Methodol. 45, 580-594. doi:10.1016/j.trb.2010.10.002

Appendix 1. For the optimal step credit scheme, equilibrium is not unique.

Proof. Denote the credit charge period as $\left[t^{+}, t^{-}\right]$, the number of credits charged as $\kappa^{c}$, and the credit demand as $\kappa^{c} s\left(t^{-}-t^{+}\right)$. Denote $\rho^{o}$ as the optimal credit price. The travel demand is given by $N=s\left(t^{-}-t^{+}\right)+s \frac{\rho^{o} \kappa^{c}}{\beta}+2 s \frac{\rho^{o} \kappa^{c}}{\alpha+\gamma}$ (Arnott et al., 1990). The credit demand is always equal to the credit supply as the number of drivers passing the bottleneck during the credit charge period is fixed. Equilibrium is not unique if there exists a credit price other than the optimal credit price such that (1) all drivers departed have the same lowest generalized travel price, and (2) the number of drivers departed is equal to the travel demand. Consider a credit price smaller than the optimal one, $\rho<\rho^{\circ}$, and assume the driver arriving at $t^{+}$ also has a queuing time, $T_{q}(t)$, which implies that he/she departs before $t^{+}$but has to wait in the queue for his/her turn to pass. The drivers arriving during the credit charge period still have the same generalized travel price as their queuing time all increases by $T_{q}(t)$. The size of mass departures after $t^{-}$will adjust based on the credit price so that the generalized travel price remains identical before and after $t^{-}$, i.e., $M=2 s \frac{\rho \kappa^{c}}{\alpha+\gamma}$. To minimize the generalized travel price, the drivers arriving earlier than $t^{+}$will adjust their departure times so that the last driver arrives just before $t^{+}$. The number of these drivers depends on the difference between $t^{+}$, i.e., $s\left(t^{+}-t_{s}\right)$. The departure time of the first driver is chosen in such a way that his/her generalized travel price is also equal to that of the driver arriving at $t^{+}$, i.e., $t_{s}=t^{+}-\frac{\alpha T_{q}+\rho \kappa^{c}}{\beta}$. In this way, all drivers departed have the same generalized travel price $p=\alpha T_{q}+\beta\left(t^{*}-t^{+}\right)+\rho \kappa^{c}$. At the same time, there always exists a $T_{q}$ such that the total number of drivers departed is equal to the travel demand, i.e., $T_{q}=\frac{\left(\rho^{o}-\rho\right) \kappa^{c}}{\alpha}+\frac{2\left(\rho^{o}-\rho\right) \beta \kappa^{c}}{(\alpha+\gamma) \alpha}$. In order to arrive at the minimal generalized travel price the price should be lower than when leaving at the end of the peak period, $t^{-}+\frac{2 \rho \kappa^{c}}{\alpha+\gamma}$, thus the credit price should satisfy $\rho>\frac{\alpha+\gamma+2 \beta}{2(\gamma+\beta)} \rho^{o}$.

To summarize: for any credit price satisfying $\frac{\alpha+\gamma+2 \beta}{2(\gamma+\beta)} \rho^{o}<\rho<\rho^{\circ}$, there always exists a $T_{q} \geq 0$ such that (1) the credit demand is equal to credit supply, (2) all drivers departed have the same lowest generalized 
travel price, and (3) the number of drivers departed is equal to the travel demand, which implies there exists a equilibrium for this credit price. Therefore, given the optimal step credit charge scheme, the resulting equilibrium is not unique. This completes the proof.

Appendix 2. For any possible equilibrium credit price $\rho$ with $\rho \geq 0$ under the tradable credit scheme given by Eqs. (18)-(19), 1). the numbers of early and late arrivals are fixed, and equal those in the optimal case, 2) the peak periods $\left[\hat{t}_{s}, \hat{t}_{e}\right]$ that have $\left\{\hat{t}_{s}<t_{s}^{\circ}, \hat{t}_{e}<t_{e}^{\circ}\right\}$, or $\left\{\hat{t}_{s}>t_{s}^{\mathrm{o}}, \hat{t}_{e}>t_{e}^{\circ}\right\}$ cannot describe an equilibrium.

Proof. Here we only consider the first case, $t_{s}<t_{s}^{0}$ and $t_{e}>t_{e}^{0}$. The generalized prices for the different time intervals satisfy the following conditions:

$$
\begin{gathered}
\beta\left(t^{*}-t^{\prime}\right)+\alpha \frac{m}{v\left(t^{\prime}\right)}=\mu, t_{s} \leq t^{\prime}<t_{s}^{o} \\
\beta\left(t^{*}-t^{\prime}\right)+\alpha \frac{m}{v\left(t^{\prime}\right)}+\rho L \beta\left(t^{\prime}-t_{s}^{o}\right)=\mu, t_{s}^{o} \leq t^{\prime} \leq t^{*} \\
\gamma\left(t^{\prime}-t^{*}\right)+\alpha \frac{m}{v\left(t^{\prime}\right)}+\rho L \gamma\left(t_{e}^{o}-t^{\prime}\right)=\mu, t^{*}<t^{\prime} \leq t_{e}^{o} \\
\gamma\left(t^{\prime}-t^{*}\right)+\alpha \frac{m}{v\left(t^{\prime}\right)}=\mu, t_{e}^{o}<t^{\prime} \leq t_{e}
\end{gathered}
$$

where $\hat{\mu}$ denotes the equilibrium travel cost satisfying $\mu=p\left(t_{s}\right)=p\left(t_{e}\right)$, and $L=v /(1+v)$. From the above conditions (A1)-(A4), given a credit price, as both the schedule delay and the credit charge are piecewise linear function of the arrival time, the travel time function must be linear as well. Combining Eqs. (12)-(13), the arrival rate function is given by:

$$
r_{A}\left(t^{\prime}\right)=\left\{\begin{array}{l}
R(\beta /(\alpha m))^{1 / v}\left(t^{\prime}-t_{s}\right)^{1 / v}, t_{s} \leq t^{\prime}<t_{s}^{o} \\
R(\beta /(\alpha m))^{1 / v}\left((1-\rho L)\left(t^{\prime}-t_{s}\right)-\rho L\left(t_{s}-t_{s}^{o}\right)\right)^{1 / v}, t_{s}^{o} \leq t^{\prime} \leq t^{*} \\
R(\gamma /(\alpha m))^{1 / v}\left((1-\rho L)\left(t_{e}-t^{\prime}\right)-\rho L\left(t_{e}^{o}-t_{e}\right)\right)^{1 / v}, t^{*}<t^{\prime} \leq t_{e}^{o} \\
R(\gamma /(\alpha m))^{1 / v}\left(t_{e}-t^{\prime}\right)^{1 / v}, t_{e}^{o}<t^{\prime} \leq t_{e}
\end{array}\right.
$$

Based on (A5), we obtain:

$$
\begin{gathered}
\int_{t_{s}}^{t^{*}} r_{A}\left(t^{\prime}\right) d t^{\prime}=R\left(\frac{\beta}{\alpha m}\right)^{1 / v} \frac{L}{1-\rho L}\left(\left((1-\rho L)\left(t^{*}-t_{s}\right)+\rho L\left(t_{s}^{o}-t_{s}\right)\right)^{1 / L}-\rho L\left(t_{s}^{o}-t_{s}\right)^{1 / L}\right) \\
\int_{t^{*}}^{t_{e}} r_{A}\left(t^{\prime}\right) d t^{\prime}=\frac{\beta}{\gamma} R\left(\frac{\beta}{\alpha m}\right)^{1 / v} \frac{L}{1-\rho L}\left(\left((1-\rho L)\left(t^{*}-t_{s}\right)+\rho L\left(t_{s}^{o}-t_{s}\right)\right)^{1 / L}-\rho L\left(t_{s}^{o}-t_{s}\right)^{1 / L}\right)
\end{gathered}
$$

Substituting the above Eqs. (A6)-(A7) into condition (16) yields:

$$
\int_{t_{s}}^{t^{*}} r_{A}\left(t^{\prime}\right) d t^{\prime}+\int_{t^{*}}^{t_{e}} r_{A}\left(t^{\prime}\right) d t^{\prime}=\Gamma\left(\left((1-\rho L)\left(t^{*}-t_{s}\right)+\rho L\left(t_{s}^{o}-t_{s}\right)\right)^{1 / L}-\rho L\left(t_{s}^{o}-t_{s}\right)^{1 / L}\right)=N
$$

where $\Gamma=(\gamma+\beta) / \gamma \cdot R(\beta /(\alpha m))^{1 / v} \cdot L /(1-\rho L)$. Therefore, the number of early arrivals is: 


$$
\int_{t_{s}}^{t^{*}} r_{A}\left(t^{\prime}\right) d t^{\prime}=\frac{\gamma}{\gamma+\beta} N
$$

which is equal to the number of early arrivals in the optimal case. Similarly, it can be proven that if $t_{s}>t_{s}^{0}$ and $t_{e}<t_{e}^{\circ}$ hold, the number of early departures is also equal to $\gamma /(\gamma+\beta) N$.

For the other two cases, where $t_{s}$ and $t_{e}$ would both be earlier or later than the optimum, it can be proven that this case does not describe an equilibrium. Here we only give the proof of the case $t_{s}>t_{s}^{0}$ and $t_{e}>t_{e}^{0}$, as the other case can be proven similarly. Denote $\hat{t}=t^{*}+\beta / \gamma\left(t^{*}-t_{s}\right)$ as the moment at which the schedule delay cost late are equal to the schedule delay cost early for the first driver. Then $\hat{t}<t_{e}^{o}$, and $m / v(\hat{t})>0$ must hold because $t_{e}>t_{e}^{\circ}$. The travel costs of drivers arriving at $t_{s}$ and $\hat{t}$ are given by:

$$
\begin{aligned}
& \beta\left(t^{*}-t_{s}\right)+\rho \kappa^{o}\left(t_{s}\right)=\beta(1-\rho L)\left(t^{*}-t_{s}\right)+\beta \rho L\left(t^{*}-t_{s}^{o}\right) \\
& \gamma\left(\hat{t}-t^{*}\right)+\rho \kappa^{o}(\hat{t})+\alpha \frac{m}{v(\hat{t})}=\beta(1-\rho L)\left(t^{*}-\hat{t}_{s}\right)+\beta \rho L\left(t^{*}-t_{s}^{o}\right)+\alpha \frac{m}{v(\hat{t})}
\end{aligned}
$$

which cannot be true as the third term in (A.11) is positive. Therefore, $t_{s}>t_{s}^{o}$ and $t_{e}>t_{e}^{0}$ cannot hold for an equilibrium. This completes the proof.

Appendix 3. The arrival rate $r_{A}\left(t^{\prime}\right)$ with $\rho>\rho^{\circ}$ displays a flatter pattern in the interval $\left[t_{s}^{0}, t^{*}\right)$.

Proof. This means the arrival rate curve $r_{A}\left(t^{\prime}\right)$ with $\rho>\rho^{\circ}$ has only one intersection with the optimal arrival rate curve $r_{A}^{o}\left(t^{\prime}\right)$ (Figure 3-1). If this is not true, and let us assume there is a second intersection for the two arrival rate curves (Figure 3-2). Denote the arrival times of the first and second intersection as $\widehat{t}$ and $\breve{t}$, respectively. Consider two arrival times $t^{\prime}$ and $t^{\prime}+\Delta t$ satisfying $\bar{t}<t^{\prime}<\breve{t}<t^{\prime}+\Delta t<t^{*}$, therefore $r_{A}\left(t^{\prime}\right)<r_{A}^{o}\left(t^{\prime}\right)$ and $r_{A}\left(t^{\prime}+\Delta t\right)>r_{A}^{o}\left(t^{\prime}+\Delta t\right)$. The generalized prices for these two arrival times with $\rho>\rho^{\circ}$ satisfy:

$$
\begin{gathered}
u\left(t^{\prime}\right)=\beta\left(t^{*}-t^{\prime}\right)+\alpha\left(T_{f}+m\left[\frac{r_{A}\left(t^{\prime}\right)}{R}\right]^{v}\right)+\hat{\rho} L \beta\left(t^{\prime}-t_{s}^{o}\right) \\
u\left(t^{\prime}+\Delta t\right)=\beta\left(t^{*}-t^{\prime}-\Delta t\right)+\alpha\left(T_{f}+m\left[\frac{r_{A}\left(t^{\prime}+\Delta t\right)}{R}\right]^{v}\right)+\rho L \beta\left(t^{\prime}+\Delta t-t_{s}^{o}\right)
\end{gathered}
$$

As every driver has the same generalized price, we have:

$$
(1-\rho L) \beta \Delta t=\alpha m\left(\left[\frac{r_{A}\left(t^{\prime}+\Delta t\right)}{R}\right]^{v}-\left[\frac{r_{A}\left(t^{\prime}\right)}{R}\right]^{v}\right)
$$

On the other hand, the generalized prices for these two arrival times with $\rho^{\circ}=1$ satisfy: 


$$
\begin{gathered}
u^{\mathrm{o}}\left(t^{\prime}\right)=\beta\left(t^{*}-t^{\prime}\right)+\alpha\left(T_{f}+m\left[\frac{r_{A}^{\mathrm{o}}\left(t^{\prime}\right)}{R}\right]^{\mathrm{v}}\right)+\rho^{\mathrm{o}} L \beta\left(t^{\prime}-t_{s}^{o}\right) \\
u^{\mathrm{o}}\left(t^{\prime}+\Delta t\right)=\beta\left(t^{*}-t^{\prime}-\Delta t\right)+\alpha\left(T_{f}+m\left[\frac{r_{A}^{\mathrm{o}}\left(t^{\prime}+\Delta t\right)}{R}\right]^{\mathrm{v}}\right)+\rho^{\mathrm{o}} L \beta\left(t^{\prime}+\Delta t-t_{s}^{o}\right)
\end{gathered}
$$

which results in:

$$
\left(1-\rho^{\mathrm{o}} L\right) \beta \Delta t=\alpha m\left(\left[\frac{r_{A}^{\mathrm{o}}\left(t^{\prime}+\Delta t\right)}{R}\right]^{\mathrm{v}}-\left[\frac{r_{A}^{\mathrm{o}}\left(t^{\prime}\right)}{R}\right]^{\mathrm{v}}\right)
$$

As $r_{A}\left(t^{\prime}\right)<r_{A}^{o}\left(t^{\prime}\right)$ and $r_{A}\left(t^{\prime}+\Delta t\right)>r_{A}^{o}\left(t^{\prime}+\Delta t\right)$, the terms on the right hand of Eq. (A14) and of Eq. (A17) satisfy:

$$
\alpha m\left(\left[\frac{r_{A}\left(t^{\prime}+\Delta t\right)}{R}\right]^{v}-\left[\frac{r_{A}\left(t^{\prime}\right)}{R}\right]^{v}\right)>\alpha m\left(\left[\frac{r_{A}^{\mathrm{o}}\left(t^{\prime}+\Delta t\right)}{R}\right]^{\mathrm{v}}-\left[\frac{r_{A}^{\mathrm{o}}\left(t^{\prime}\right)}{R}\right]^{\mathrm{v}}\right)
$$

On the other hand, $\rho>\rho^{\circ}$ implies that the terms on the left hand of Eq. (A14) and of Eq. (A17) satisfy:

$$
(1-\rho L) \beta \Delta t<\left(1-\rho^{\circ} L\right) \beta \Delta t
$$

As the term on the right hand is equal to the term on the left hand in both Eq. (A14) and Eq. (17), Eqs. (A18)-A(19) are inconsistent. This completes the proof.

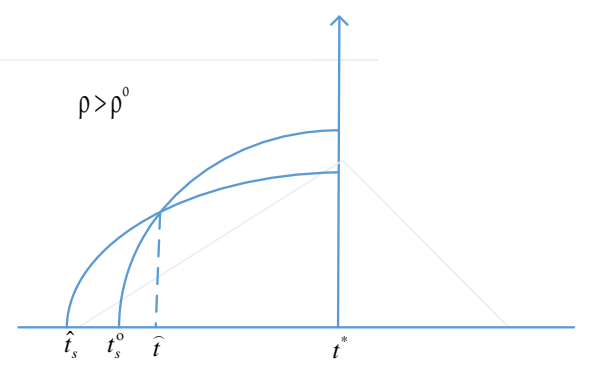

Figure 3-1. The arrival rate curves with one intersection

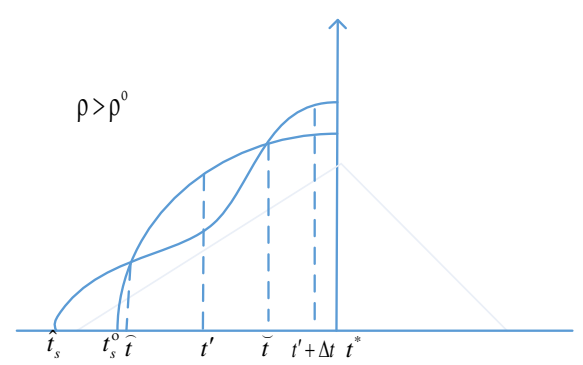

Figure 3-2. The arrival rate curves with two intersections

Figure 3. The intersections of the arrival rate curves for credit price $\rho>\rho^{0}$ and for the optimum

Appendix 4. A departure time based optimal credit charge scheme cannot lead to a unique equilibrium in the bottleneck model.

Proof. We use the Vickrey's bottleneck model to verify the existence of the multiple equilibria under the optimal tradable credit scheme. Define the following optimal credit charge rate based on departure time as below 


$$
\kappa^{\mathrm{so}}(t)=\left\{\begin{array}{l}
\beta\left(t-t_{s}^{\mathrm{so}}\right), t_{s}^{\mathrm{so}} \leq t \leq t^{*} \\
\gamma\left(t_{e}^{\mathrm{so}}-t\right), t^{*}<t \leq t_{e}^{\mathrm{so}}
\end{array}\right.
$$

where $t$ denote the departure time. The amount of credit issued is given by:

$$
\mathrm{K}^{\mathrm{so}}=\int_{t_{s}^{\mathrm{so}}}^{t_{e}^{\mathrm{so}}} s \kappa(t) d t=\frac{\gamma \beta}{2(\gamma+\beta)} \frac{N^{2}}{s}
$$

Given the above tradable credit scheme (A20)-(A21), if the peak period is the same as the credit charge period, the departure rate is given by:

$$
r_{D}(t)=\left\{\begin{array}{l}
\frac{\alpha-\rho \beta}{\alpha-\beta} s, t_{s}^{\mathrm{so}} \leq t \leq \tilde{t} \\
\frac{\alpha-\rho \beta}{\alpha+\gamma} s, \tilde{t}<t \leq t^{*} \\
\frac{\alpha+\rho \gamma}{\alpha+\gamma} s, t^{*}<t \leq t_{e}^{\mathrm{so}}
\end{array}\right.
$$

If the credit price is equal to 1 , the resulting equilibrium is socially optimum and the amount of credits consumed, $\mathrm{K}$, is equal to the amount of credit issued, $\mathrm{K}^{\text {so }}$. Now, we further analyze that if there exists another equilibrium. Assume the credit price is zero, the departure rate function is then given by:

$$
r_{D}(t)=\left\{\begin{array}{l}
\frac{\alpha}{\alpha-\beta} s, t_{s}^{\mathrm{so}} \leq t \leq \tilde{t} \\
\frac{\alpha}{\alpha+\gamma} s, \tilde{t}<t \leq t_{e}^{\mathrm{so}}
\end{array}\right.
$$

where $\tilde{t}=t^{*}-\beta \gamma / \alpha(\beta+\gamma) \cdot N / s$. The amount of credit consumed can be calculated as:

$$
\mathrm{K}=\frac{\alpha s}{\alpha-\beta} \int_{t_{s}^{\mathrm{so}}}^{\tilde{t}} \kappa(t) d t+\frac{\alpha S}{\alpha+\gamma} \int_{\tilde{t}}^{t_{e}^{\mathrm{so}}} \kappa(t) d t=\frac{\alpha \beta s}{2(\alpha-\beta)}\left(\tilde{t}-t_{s}^{\mathrm{so}}\right)^{2}+\frac{\alpha \gamma s}{2(\alpha+\gamma)}\left(t_{e}^{\mathrm{so}}-\tilde{t}\right)^{2}=\frac{\gamma \beta}{2(\gamma+\beta)} \frac{N^{2}}{s}
$$

which is also equal to the amount of credit issued, $\mathrm{K}^{\text {so }}$. Therefore, all the equilibrium conditions are satisfied and the departure rate (A23) is also an equilibrium departure, implying non-uniqueness. Therefore, even if the credit demand function depends on the variable departure rate, the resulting equilibrium is still non-unique. This completes the proof.

\section{Acknowledgement}

This work is supported by funding of the Netherlands Organization for Scientific Research (NWO) as part of the U-SMILE project 438-15-176, which is gratefully acknowledged. 